\title{
From Static to Dynamic Modeling of Surfactants Micellization
}

Micelles and micellization appeal long lasting interest as promising drug carriers. A conventional parameter providing information about formation of micelles is critical micelle concentration (cmc). Its value roughly separates two states of the surfactant solution - namely states with and without presence of micelles. If concentration of surfactants in water solution approaches $\mathrm{cmc}$ some physical quantities abruptly change, and this phenomenon is a key to determine value of the $\mathrm{cmc}$. From numerous approaches for determination of the $\mathrm{cmc}$ the paper considers the conductivity-based method. But rather than studying the mechanism of micellization that is primarily carried out by the colloid chemists, the paper is focused on the development of an information rich and optimal dynamical model of the conductivity vs. concentration dependence. The model is derived from the solutions of the $1^{\text {st }}$ order differential equation. The optimal model parameters are determined by the downhill simplex algorithm and the $\mathrm{cmc}$ is computed on the basis of the curvature of the concentration dependence of the conductivity.

Key words: Modeling, optimization, conductivity, micellization

\begin{abstract}
Von der statischen zur dynamischen Modellierung der Mizellenbildung von Tensiden. Mizellen und Mizellenbildung erfahren als vielversprechende Medikamententräger ein lang anhaltendes Interesse. Ein herkömmlicher Parameter, der Informationen über die Bildung von Mizellen liefert, ist die kritische Mizellenkonzentration $(\mathrm{cmc})$. Sein Wert trennt grob zwei Zustände der Tensidlösung, nämlich Zustände mit und ohne Vorhandensein von Mizellen. Wenn sich die Konzentration von Tensiden in wässriger Lösung dem cmc-Wert nähert, ändern sich einige physikalische Größen schlagartig. Dieses Phänomen ist ein Schlüssel zur Bestimmung des cmc-Wertes. Aus zahlreichen Ansätzen zur Bestimmung der $\mathrm{cmc}$ wird im Beitrag die leitfähigkeitsbasierte Methode betrachtet. Anstatt jedoch den Mechanismus der Mizellenbildung zu untersuchen, der in erster Linie von den Kolloidchemikern durchgeführt wird, konzentriert sich das Papier auf die Entwicklung eines informationsreichen und optimalen dynamischen Modells der Abhängigkeit der Leitfähigkeit von der Konzentration. Das Modell wird aus den Lösungen der Differentialgleichung 1. Ordnung abgeleitet. Die Parameter des optimalen Modells werden mit Hilfe des Downhill-Simplex-Algorithmus bestimmt und die $\mathrm{cmc}$ wird auf der Grundlage der Krümmung der Abhängigkeit "Leitfähigkeit vs. Konzentration" berechnet.
\end{abstract}

Institute of Robotics and Cybernetics, Faculty of Electrical Engineering and Information Technology, Ilkovičova 3, 81219 Bratislava, Slovak Republic

Department of Physical Chemistry of Drugs, Faculty of Pharmacy, Comenius University in Bratislava, Odbojárov 10, 83232 Bratislava, Slovak Republic
Stichwörter: Modellierung, Optimierung, Leitfähigkeit, Mizellisierung

\section{Introduction}

Surfactants (surface active substances) are composed of hydrophilic polar head group and nonpolar hydrophobic tail group. For instance, molecules of ionic surfactants have relatively large nonpolar part (a long aliphatic chain) and smaller polar part. Due to this, if the concentration of surfactant in the water solution approximates a certain value its molecules start to self-assemble into the formations of size less than $50 \mathrm{~nm}$ that are known as micelles [1]. The illustrating example in the end of the paper uses results of the studies of the ionic surfactants. In accordance with [1] the critical micelle concentration $(\mathrm{cmc})$ is given by a small range of concentration of the surfactant below which virtually no micelles are detected and above which virtually all additional surfactant molecules form the micelles. More precise is the Philip's definition [2], which in essence states: The cmc is a concentration of surfactant for which the maximal rate of the appearance of new micelles, i. e. $\max (d f(c) / d c)$ is reached, where $f$ means the actual number of micelles in water solution. This verbal definition amounts to fulfilling the following mathematical condition $d^{3} f(c) / d c^{3}=0$.

Processes of the self-assembling are invoked by the hydrophobic interactions between hydrocarbon parts of surfactant molecules and electrostatic repulsive forces. In comparison with nonionic surfactants the higher concentration is required to overcome the electrostatic repulsion forces between ionic head groups. A direct consequence is that the ionic surfactants of the same alkyl chain length are more difficult to aggregate and their cmc values are some orders of magnitude larger [2].

Simultaneously with the appearance of micelles the changes of some physical properties of the solution, like the osmotic pressure, surface tension, electric conductivity and others can be observed. Just this phenomenon opens the doors for the development of various methods of establishing the cmc.

In view of the topic of this paper it is important to note that the process of self-assembling is not instantaneous but, as any other dynamical process, it takes some time. If concentration of surfactants in solution increases and reaches a value close to the $\mathrm{cmc}$, one can observe the gradual onset of the self-assembling. If the rate of the process reaches a peak value it gradually decreases towards a steady-state rate. Hence; around the $\mathrm{cmc}$ is a separating concentration range within which the maximal rate of change of the chosen physical property is reached. 
The dependence of conductivity above and below of the separating range is commonly approximated by the straight lines of different slopes. Therefore, the $1^{\text {st }} \mathrm{cmc}$ is commonly (and relatively correctly) determined by the intersection of the approximating lines. But the drawback of such a linear approximation is evident. It provides nearly no information about the rate of micellization. That is why this paper intends to present a kind of the dynamic approach to evaluate of the conductivity $(\kappa)$ vs. concentration (c) dependence, i.e. $\kappa=f(c)$, which should be able to express both the strength and rate of the process of micellization. The instantaneous strength will be indicated by the instantaneous conductivity while the rates of micellization by the instantaneous rate of the conductivity change.

Note that some surfactant may also exhibit the $2^{\text {nd }}$ and even $3^{\text {rd }} \mathrm{cmc}$. If the concentration of a surfactant exceeds the $1^{\text {st }} \mathrm{cmc}$ by a factor 2 to 10 for some single-chained ionic surfactants, including those the authors have studied in [3], the second gradual increase of the number of newly created micelles appeared. The shapes of these new micelles may no longer follow the spherical shape $[4,5]$. The second peak of the rate of micellization is shown in Figs. 1 and 2 and the corresponding concentration is just the $2^{\text {nd }} \mathrm{cmc}$.

The first objective of this study consists in describing the dependence $\kappa=f(c)$ as a static relation in two forms. The first may be called as a descriptive form while the second is an IF-THEN rules form. The second objective consists in describing the dependence $\kappa=f(c)$ dynamically in the form of a particular solution of the $1^{\text {st }}$ order differential equation. To this end the dynamic model will be synthesized and its parameters will be optimized by the computer simulation. An advantage of the dynamic description rests in its ability to capture the continual transition through the separating range, i.e. from the totally non-micellized state to (virtually) fully micellized state as a truly dynamic process. A natural way of doing this consists in finding an appropriate solution of the dynamic model - the differential equation. The third objective consists in demonstrating the obtained results by an example. The theory developed here will be verified on the results of the measurements the authors obtained early [3].

rate of creation

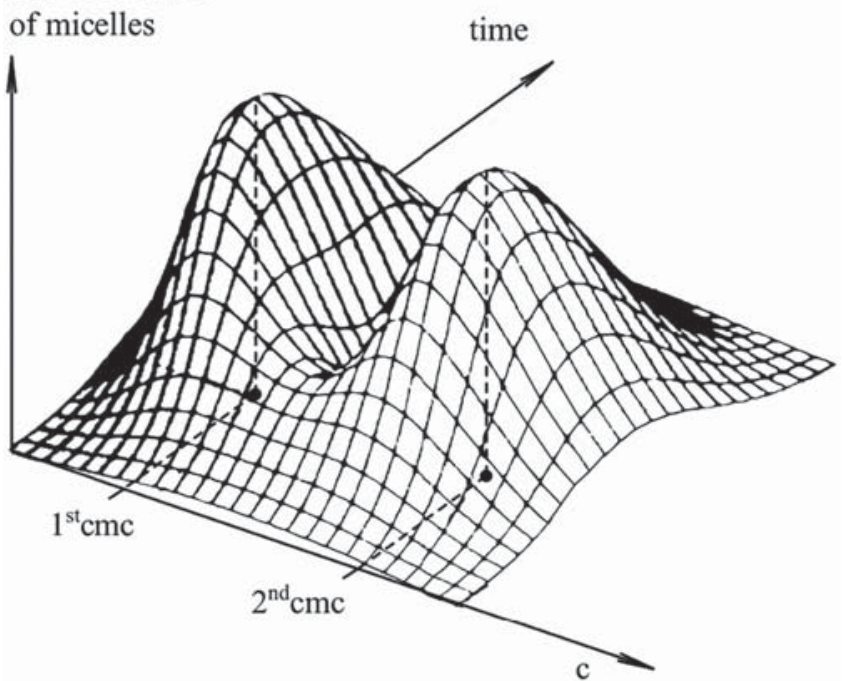

Figure 1 The process rate as a function of concentration and time
Before doing this let us first assume that the (total) concentration of the surfactant in the water solution increases. At the moment the concentration reaches values that are close to the $1^{\text {st }} \mathrm{cmc}$ the rate of the creation of new micelles start to gradually increase as far as it reaches a peak value. At this time instant the rate starts to gradually decrease towards a certain steady-state rate, which is given by the slope of line between the $1^{\text {st }}$ and the $2^{\text {nd }} \mathrm{cmc}$. Hence; the rates of the self-assembling and the rate of the conductivity change of the solution are dynamic processes and as such they can be described by a differential equation. The term "rate" is here exclusively used in relation to the time. In what follows, for the speed by which the conductivity changes with respect to the (changing) concentration we use the term "gradient".

The Fig. 1 portrays the process rate $(\mathrm{r})$ as a function of two parameters - concentration and time, i.e. $r=f(c, t)$. Values of the both cmc data are given by the peaks of the 3D graph. The rate of micellization is depicted as a function of two variables - the concentration and time. The illustration emphasizes that the process is dynamic and smooth rather than static process that undergoes an abrupt change. The created micelles have spherical shapes and the packing parameter is $<0: 33$ [4].

As follows from the above explanations, the full range of concentrations consists of two linear segments that are separated by a narrow separating interval shown in Fig. 2 in which the most molecules are micellized. The first segment extends over the interval $0-1^{\text {st }} \mathrm{cmc}$ where virtually no micelles exist while the second one extends over the interval $1^{\text {st }} \mathrm{cmc}-2^{\text {nd }} \mathrm{cmc}$ where virtually all surfactants' molecules are micellized.

Some authors try to approximate the conductivity function over the separating interval (Fig. 2) by parametric statistically based approaches. For instance in [6] the $1^{\text {st }} \mathrm{cmc}$ is determined as the mean value of the Gauss curve. This implies that the separating interval is symmetrical with respect to the $1^{\mathrm{s}} \mathrm{cmc}$, which may not be always true (!). Rather, the onset of the appearance of micelles is more rapid than its fading. The authors observed this phenomenon in virtually all experiments aimed at the determination of the $\mathrm{cmc}$ [3].

Another approximation of the regression function may be based on nonparametric approaches. For instance in [7] no

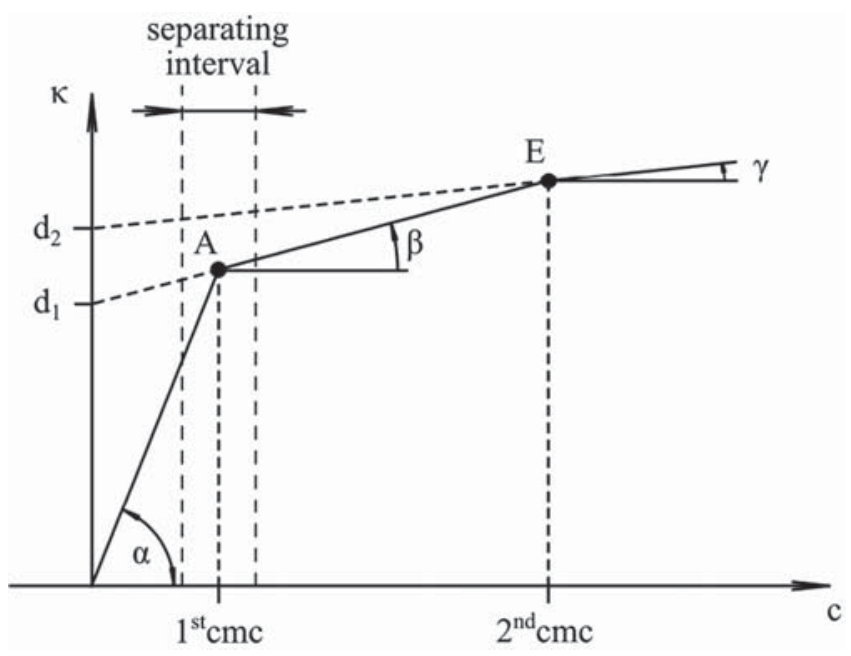

Figure 2 The typical dependence $\kappa=f(c)$ 
structure of the (nonlinear) regression function is supposed. It may be approximated either deterministically (for instance by the spline approximation) or statistically. Here it is worth mentioning that the parametric and nonparametric approaches are complementary rather than antagonistic and each has its pros and cons. Nonparametric approaches are more universal but computationally demanding, while the estimation of parametric models is reduced to a few parameters which make them very efficient. In this paper is adopted the parametric but dynamic approach.

While the micelles appearing in the vicinity of the $1^{\text {st }} \mathrm{cmc}$ are of the spherical shape, those appearing at and above the $2^{\text {nd }} \mathrm{cmc}$ are road - like shaped. [4] It means that the $2^{\text {nd }} \mathrm{cmc}$ is connected with some structural transitions. In relation to the $2^{\text {nd }} \mathrm{cmc}$ the authors share the opinion presented in [4] which says: "The term $2^{\text {nd }} \mathrm{cmc}$ should be treated with some caution" because the second brake in the conductivity vs. concentration dependence may not be invoked by transition of the spherical to the prolonged micelles, but rather by the interionic and inter micellar interactions. That is also reason for existence of small curvature of the plot above the $1^{\text {st }} \mathrm{cmc}$, though it is commonly approximated by the linear segment. It is also known that more distinct structural transition may be induced by adding inorganic electrolytes or some nonelectrolytes.

In conclusion it is worth mentioning that the rate of micellization has also the strong impact on the development of the advanced dosage forms. The fact is used that micelles may entrap the drug molecule. Hence, it may be said that the higher the rate of micellization the lower the rate of bioavailability and also the lower the rate of the therapeutic performance. This expert knowledge is intensively utilized in the development of the controlled and targeted delivery of the drugs with an ultralow cmc $[8,9]$.

\section{Some preliminaries models of micellization}

Typical dependence of conductivity vs. concentration consists of the straight-line segments as shown Fig. 2.

Physical reasons behind Fig. 2 may be formulated in the following way: Due to the presence of a sufficient number of free ions the conductivity initially increases linearly with increasing concentration. When the concentration approaches the $1^{\text {st }} \mathrm{cmc}$ one can observe the quick onset of the newly emerging micelles. Immediately after that virtually all surfactants molecules are micellized and new micelles appears only due to further delivery of new surfactant molecules.

At this situation the movability of free ions is hampered by prevailing number of the micellized molecules. Therefore, the conductivity increases further but with the shrinking rate, which relatively quickly approaches a constant rate. That is why the dependence conductivity vs. concentration is commonly (and roughly) modelled with the straight-line segments as shows Fig. 2 and the $1^{\text {st }} \mathrm{cmc}$ is determined by intersection of two regressing lines.

A drawback of such a linear portrait is that the immanent dynamic of the process is totally omitted. Therefore real behaviour of the process of micellization evokes the researcher to model the dependence of conductivity vs, concentration by a suitable nonlinear function. In this paper we suggest to smooth the dependence not only in the vicinity of the $1^{\text {st }} \mathrm{cmc}$ as it is done in [6], but we smooth the whole dependence.

\section{The static model}

The conductivity $\kappa(\mathrm{c})$ over the whole concentration interval can be approximated by three straight line segments: 0-A, $A-E$, and over $E$ with the slopes $k_{1}=\operatorname{tg}(\alpha), k_{2}=\operatorname{tg}(\beta)$ and $\mathrm{k}_{3}=\operatorname{tg}(\gamma)$ respectively. The intersection of the first two lines defines point $\mathrm{A}$ and the intersection of the second and third line defines point $\mathrm{E}$. The conductivity $\kappa(\mathrm{c})$ over the interval $0-1^{\text {st }} \mathrm{cmc}$ is described by the equation:

$\kappa(\mathrm{c})=\mathrm{k}_{1}\left[\mathbf{1}(\mathrm{c})-\mathbf{1}\left(\mathrm{c}-1^{\mathrm{st}} \mathrm{cmc}\right)\right] \mathrm{c} \cdot \mathrm{k}_{1}=\operatorname{tg}(\alpha)$

The function 1(c) is known as the Heaviside function [11]. It is conventionally written in bolt, which stresses that $\mathbf{1}$ (c) is a function of the independent variable " $c$ "and not the mere number one. It is defined by the equation (2).

$\mathbf{1}(\mathrm{c})=0$ for $\mathrm{c}<0$ and $\mathbf{1}(\mathrm{c})=1$ for $\mathrm{c} \geq 0$

The expression $1\left(\mathrm{c}-1^{\mathrm{st}} \mathrm{cmc}\right)$ in (1) means that the function $\mathbf{1}(\mathrm{c})$ is shifted along the positive axis by $1^{\text {st }} \mathrm{cmc}$ units. The geometrical interpretation of the whole expression [1(c)$\left.1\left(\mathrm{c}-1^{\text {st }} \mathrm{cmc}\right)\right]$ in $(1)$ is a rectangle over the line segment $0-1^{\text {st }}$ cmc as shows the thick line in Fig. 3.

The linear increase of the conductivity $\kappa(c)$ over the interval $\left(1^{\text {st }} \mathrm{cmc}-2^{\text {nd }} \mathrm{cmc}\right)$ is approximated by the equation (3).

$\kappa(\mathrm{c})=\mathrm{k}_{2}\left[\mathbf{1}\left(\mathrm{c}-1^{\mathrm{st}} \mathrm{cmc}\right)-\mathbf{1}\left(\mathrm{c}-2^{\mathrm{nd}} \mathrm{cmc}\right)\right] \mathrm{c}+\mathrm{d}_{1} \mathrm{k}_{2}=\operatorname{tg}(\beta)(3)$

Analogously, the conductivity of the solution for concentrations greater than $2^{\text {nd }} \mathrm{cmc}$ is given by the equation:

$\kappa(c)=k_{3}\left[1\left(c-2^{\text {nd }} c m c\right)\right] c+d_{2} \quad k_{3}=\operatorname{tg}(\beta)$

Finally, the mathematical expression of the conductivity over the whole concentration interval can be approximately described as the sum of linear segments:

$$
\begin{aligned}
& \kappa(\mathrm{c})=\mathrm{k}_{1}\left[\mathbf{1}(\mathrm{c})-\mathbf{1}\left(\mathrm{c}-1^{\mathrm{st}} \mathrm{cmc}\right)\right] \mathrm{c}+\mathrm{k}_{2} \\
& {\left[\mathbf{1}\left(\mathrm{c}-1^{\text {st }} \mathrm{cmc}\right)-\mathbf{1}\left(\mathrm{c}-2^{\text {nd }} \mathrm{cmc}\right)\right] \mathrm{c}+\mathrm{d}_{1}+} \\
& \mathrm{k}_{3}\left[\mathbf{1}\left(\mathrm{c}-2^{\text {nd }} \mathrm{cmc}\right)\right] \mathrm{c}+\mathrm{d}_{2}
\end{aligned}
$$

\section{The IF-THEN form of a static model}

An idea behind the process of the micellization is as follows: For concentrations below the $1^{\text {st }} \mathrm{cmc}$ no micelles exist but above it

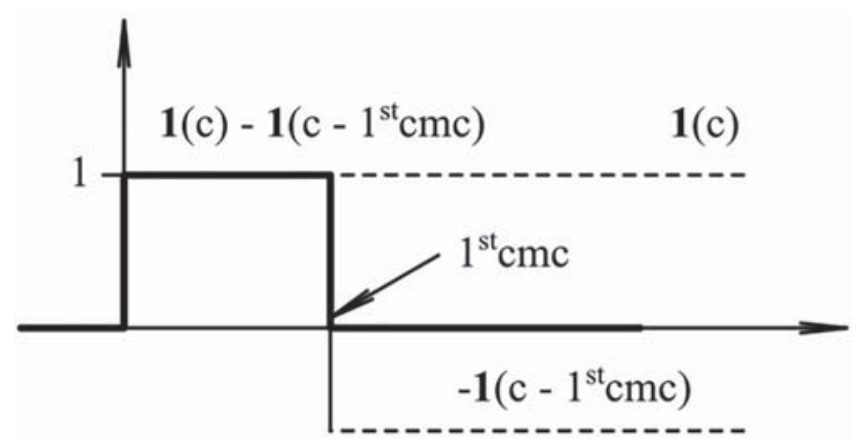

Figure 3 The function $1(\mathrm{c})$ 
the surfactant is supposedly (!) completely micellized. Hence, the information imbedded in models (3) - (5) is rather pure.

Slightly greater piece of information is imbedded in the IF-THEN rule model, which distinguishes between the total, monomeric and micellized concentrations of surfactants. Let us denote the total (monomeric + micellized) concentration of the surfactant as $c$, the concentration of monomeric surfactant as $c_{1}$ and the concentration of micellized surfactant as $c_{m}$. Then the rough but easily implementable model describing both conductivities and concentrations in terms of $c_{1}, c_{m}$ is below. The similar though significantly simplified model can be found in [12].

IF $\mathrm{c}<1^{\text {st }} \mathrm{cmc}$ THEN $\mathrm{c}_{1} \approx \mathrm{c}$ and $\mathrm{c}_{\mathrm{m}}=0$ and $\kappa=\mathrm{k}_{1} \mathrm{c}$

IF $\mathrm{c} \approx 1^{\text {st }} \mathrm{cmc}$ THEN $\mathrm{c}_{1} \approx \mathrm{c}_{\mathrm{m}} \approx 1^{\text {st }} \mathrm{cmc}$ and $\kappa=\kappa\left(1^{\text {st }} \mathrm{cmc}\right)$

IF $1^{\text {st }} \mathrm{cmc}<\mathrm{c}<2^{\text {nd }} \mathrm{cmc}$ THEN $\mathrm{c}_{1} \approx 0$ and $\mathrm{c}_{\mathrm{m}} \approx \mathrm{c}$ and $\kappa=\mathrm{k}_{2} \mathrm{c}+\mathrm{d}_{1}$ only globe-like micelles exist

IF $\mathrm{c} \approx 2^{\text {nd }} \mathrm{cmc}$ THEN $\mathrm{c}_{1} \approx 0$ and $\mathrm{c}_{\mathrm{m}}=2^{\text {nd }} \mathrm{cmc}$ and $\kappa=\kappa\left(2^{\text {nd }} \mathrm{cmc}\right)$ onset of rod-like micelles

IF $\mathrm{c}>2^{\text {nd }} \mathrm{cmc}$ THEN $\mathrm{c}_{1} \approx 0$ and $\mathrm{c}_{\mathrm{m}}=\mathrm{c}$ and $\kappa=\mathrm{k}_{3} \mathrm{c}+\mathrm{d}_{2}$ only rod like micelles exist

The sign $\approx$ means "approximately equal" and $\mathrm{k}_{1}, \mathrm{k}_{2}$ are slopes of the linear segments.

\section{The dynamic model}

Based on the physical opinion and in the congruence with the everyday experience one can reasonably expect that the appearance of micelles is a dynamical process. It means that all micelles cannot appear at the same time instant. Let us remind here the old philosophical wisdom "Natura non facit saltus" (Nature does not make jumps). Transitions of the conductivities $\kappa(\mathrm{c})$ from one linear segment to another cannot happen abruptly - they should be smooth. It means that the process of micellization should exhibit its own dynamics. Examples of the natural dynamical processes are radioactive decays, charging/discharging capacitors, growth of bacteria, growth of trees, and growth of animals to mention only few.

In this regard the aim of paper is to synthetize a model of dynamic behavior of the dependence conductivity vs. concentration. Contrary to the work of Garcia-Mateos [6], who conceived the transitions from the non-micellized to micellized state from the perspective of the probability theory. In particular, in the role of the approximation function they used the Gauss function, while this study has adopted the deterministic view. While the approach of Garcia - Mateos et al. estimates the spread of the conductivity values

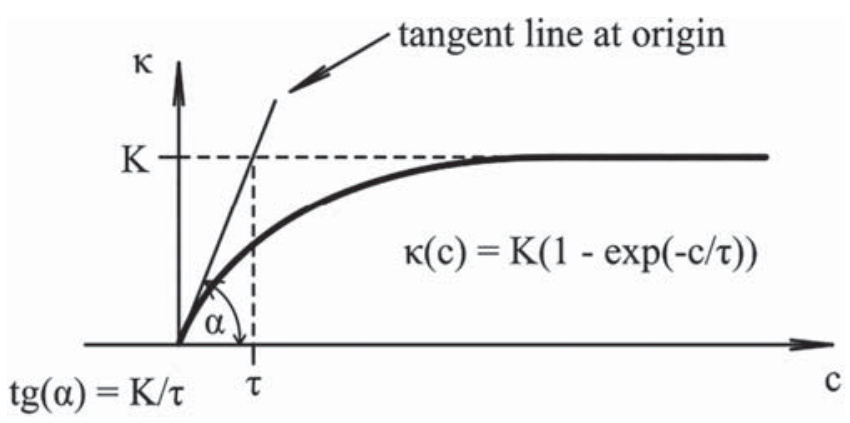

Figure 4 The function $\kappa(\mathrm{c})$ around the $1^{\text {st }} \mathrm{cmc}$ by the standard deviation, the approach presented here estimates it dynamically - in the form of a solution of a differential equation. It does not require any involving computations; it is natural and provides sufficient and verifiable information about transitions between the neighboring linear segments. Inspired by the system theory, the authors have decided to approximate the piecewise linear dependence of the conductivity vs. concentration (shown in Fig. 2), by the continuous function, namely by the solution of the $1^{\text {st }}$ order differential equation (6). Such a function approximates behavior of the conductivity $\kappa(\mathrm{c})$ not only in the vicinity of the $1^{\text {st }} \mathrm{cmc}$ but also in all concentrations between the point $\mathrm{B}$ and the point $\mathrm{E}$ as it is shown in Fig. 5 by the thick curve. A meaning of the point B will be specified in the sequel.

The approach adopted in this paper is partially inspired by the system theory; therefore, we borrow and briefly explain some terminology from this theory. The equation (6) is a conceived as a mathematical model of a dynamical system - in particular, the $1^{\text {st }}$ order linear time invariant dynamical system [12]. The time as the independent variable is replaced by the concentration. The input to the system is a constant quantity $\mathrm{K}$. In this specific case the constant $\mathrm{K}$ has the meaning of a static gain of the system, that is the steady state value of the solution to equation (6) for the input $\mathrm{K}=1$.

$\tau \mathrm{d} \kappa / \mathrm{dc}+\kappa(\mathrm{c})=\mathrm{K}$ with an initial conductivity $\kappa(\mathrm{c}=0)=0$

As it will follow from the equation (7) and Fig. 4, the steady state value $\mathrm{K}$ of the conductivity function $\kappa(\mathrm{c})$ is equal to the limit value of the function $\kappa(c)$ if the concentration approaches a value of infinity. Similarly, in the system sense, the parameter $\tau$ is so-called time constant, because it is a measure of the speed by which the system responses to an input excitation. The smaller the $\tau$ is the quicker is the system's response. But in the context of the problem resolved in this paper we abandon the terms static gain and time constant. We will simply call them as parameters $\mathrm{K}$ and $\tau$. The parameter $K$ is equal to the steady state value

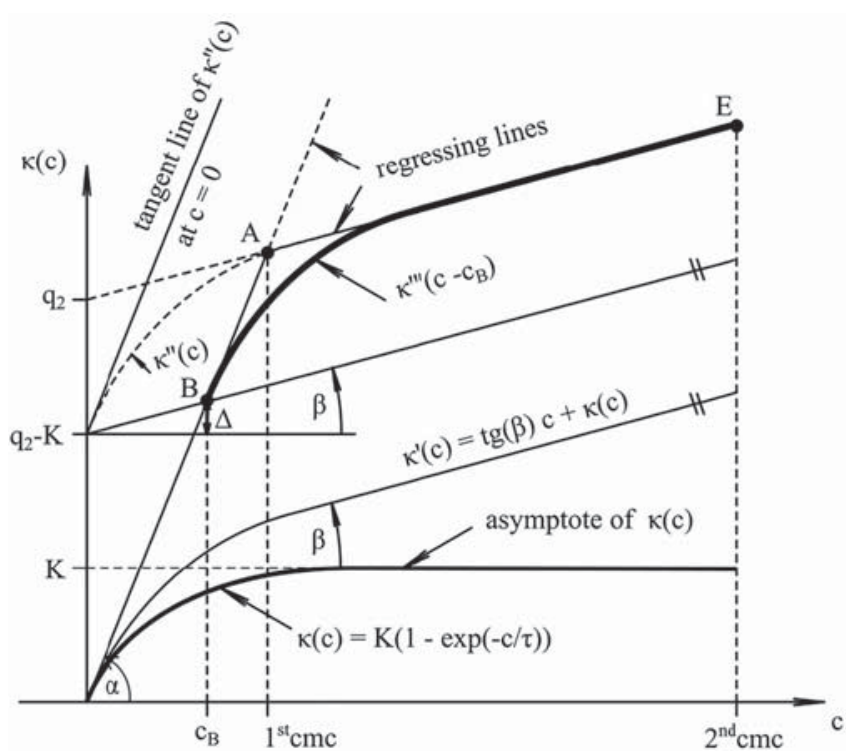

Figure 5 Steps of optimization procedure 
of the function (7) and $\tau$ tells us how quickly the conductivity function $\kappa(\mathrm{c})$ will approach its asymptote, which in this case, is the line connecting point $\mathrm{A}$ and $\mathrm{E}$. The analytic solution of the equation (6) for the initial condition $\kappa(\mathrm{c}=0)=0$ is given by the nonlinear function (7).

$\kappa(c)=K(1-\exp (-\mathrm{c} / \tau))$

One can easily compute that the concentration $c=\tau$ is needed for the conductivity $\kappa$ to reach $63.2 \%$ of its steady state value, $c=2 \tau$ for reaching $86.5 \%, c=3 \tau$ for reaching $95 \%$, and finally c $=4 \tau$ for reaching $98.3 \%$ of its steady state [11]. Therefore, it may be reasonable to suppose that for $c \geq 3 \tau$ the terminal part of the conductivity $\kappa(c)$ will virtually exactly approximate the straight line defined by the line segment A-E. Hence, the constant $\tau$ provides sufficiently rich information about the dynamical behavior of $\kappa(\mathrm{c})$. The graph of $\kappa(\mathrm{c})$ is shown in Fig. 4 and it shows that the numerical value of the constant $\tau$ is equal to the concentration needed for conductivity to reach its steady state value if it would increase along the tangent line constructed in the origin to the dependence $\kappa(\mathrm{c})$. Clearly, the straight line $\kappa(\mathrm{c})=\mathrm{K}$ is an asymptote to the function $\mathrm{k}(\mathrm{c})$.

\subsection{Optimal approximation of $\kappa(\mathrm{c})$}

Here we will present the general procedure of the optimal approximation of the measured dependence $\kappa(\mathrm{c})$ in the four steps.

Firstly, make the asymptote of $\kappa$ (c) shown in Fig. 5 to be parallel to the line defined by points $\mathrm{A}$ and $\mathrm{E}$. There are two possibilities - either rotate function (7) around the origin by the angle $\beta$ or superimpose it on the slanted line $\kappa(c)=\operatorname{tg}(\beta) c$ (not depicted in Fig. 5). Though the second possibility slightly modifies the original meaning of the constant $\tau$, it does not change the scale of the concentration axis what simplifies the whole procedure. Therefore, we adopted the second possibility. After superimposing $\kappa(\mathrm{c})$ on the line $\kappa(c)=\operatorname{tg}(\beta) c$ we obtain the function $\kappa^{\prime}(\mathrm{c})$ :

$\kappa^{\prime}(\mathrm{c})=\operatorname{tg}(\beta) c+\kappa(c)$

The mutually parallel lines are stressed by the symbol \|. Before proceeding further, it would be worth reminding the following result from the planar geometry: For the straight line $y=p x+q$, to be an asymptote of a function $f(x)$, its parameters $\mathrm{p}$, q must take the following values:

$\mathrm{p}=\lim _{\mathrm{x} \rightarrow \infty}[\mathrm{f}(\mathrm{x}) / \mathrm{x}]$

$\mathrm{q}=\lim _{\mathrm{x} \rightarrow \infty}[\mathrm{f}(\mathrm{x})-\mathrm{px}]$

Taking it into account, the asymptote of the function (8) should be given by the straight line (10)

$\kappa(\mathrm{c})=\mathrm{pc}+\mathrm{q}$

with the following parameters:

$\mathrm{p}=\lim _{\mathrm{c} \rightarrow \infty}[[\operatorname{tg}(\beta) \mathrm{c}+\mathrm{K}(1-\exp (-\mathrm{c} / \tau))] / \mathrm{c}]=\operatorname{tg}(\beta)$

$\mathrm{q}=\lim _{\mathrm{c} \rightarrow \infty}[\operatorname{tg}(\beta) \mathrm{c}+\mathrm{K}(1-\exp (-\mathrm{c} / \tau))-\operatorname{tg}(\beta) \mathrm{c}]=\mathrm{K}$

Secondly, shift the graph $\kappa^{\prime}(\mathrm{c})$ in the positive vertical direction to the point $\mathrm{E}$, whose horizontal distance from the point $\mathrm{A}$ is sufficiently large. For instance, (if the $2^{\text {nd }} \mathrm{cmc}$ is known), the point $\mathrm{E}$ may be a point representing the conductivity $\kappa(c)$ at the $2^{\text {nd }} \mathrm{cmc}$ as it is shown in Fig. 5. The shifted function is denoted by $\kappa^{\prime \prime}(\mathrm{c})$. Such a choice will secure that the value of $\kappa^{\prime \prime}(\mathrm{c})$ at the point $\mathrm{E}$ will coincide with its asymptote. As to any function cannot come in the contact with its own asymptote, this operation introduce a small incorrectness, but from the practical aspect it is quite acceptable. This contention is substantiated by the fact that for concentration $c=5 \tau$ the $\kappa(c)$ given by $(7)$ reaches $99.3 \%$ of its steady state or, in the other words, it approximates its own asymptote with less than $1 \%$ error. Clearly, as Fig. 5 shows the graph of $\kappa^{\prime}(\mathrm{c})$ should be shifted upward by the distance:

$\mathrm{d}=\left(\mathrm{q}_{2}-\mathrm{K}\right)$

The shifted graph $\kappa^{\prime \prime}(\mathrm{c})$ has, with regard to (8), the analytic expression of the form (13).

$\kappa^{\prime \prime}(\mathrm{c})=\operatorname{tg}(\beta) \mathrm{c}+\kappa(\mathrm{c})+\mathrm{d}$

Thirdly, find the derivation of $\kappa^{\prime \prime}(\mathrm{c})$ with respect to $\mathrm{c}$ :

$\mathrm{d}\left(\kappa^{\prime \prime}(\mathrm{c})\right) / \mathrm{dc}=\operatorname{tg}(\beta)+(\mathrm{K} / \tau) \exp (-\mathrm{c} / \tau)$

Thus, for the slope of the tangent line at $\mathrm{c}=0$ we will get:

$\left[\mathrm{d}\left(\kappa^{\prime \prime}(\mathrm{c})\right) / \mathrm{dc}\right]_{\mathrm{c}=0}=\operatorname{tg}(\beta)+(\mathrm{K} / \tau)$

Therefore, the tangent line of $\kappa^{\prime \prime}(c)$ at $c=0$ is expressed by the equation:

$\kappa(\mathrm{c})=[\operatorname{tg}(\beta)+(\mathrm{K} / \tau] \mathrm{c}+\mathrm{d}$

Let us remind, that the sense of mathematical operations, we have performed so far, follows the main aim of the paper, that is to replace the abrupt change of the conductance $\kappa(\mathrm{c})$ at the point A by the nonlinear smooth curve $\kappa^{\prime \prime}(\mathrm{c})$ given by the equation (13) or more precisely by its shifted version $\kappa^{\prime \prime \prime}\left(\mathrm{c}-\mathrm{C}_{\mathrm{B}}\right)$, which is driven by the thick curve in Fig. 5.

To obtain the analytical expression for $\kappa^{\prime \prime \prime}\left(\mathrm{c}-\mathrm{C}_{B}\right)$ we should find a point $B$ - the point where the curve $\kappa^{\prime \prime \prime \prime}(c)$ leaves the regression line $\kappa(c)=\operatorname{tg}(\alpha) c+q_{1}$. The graph of $\kappa^{\prime \prime \prime}\left(\mathrm{c}-\mathrm{C}_{\mathrm{B}}\right)$ lies partially on the $\kappa^{\prime \prime}(\mathrm{c})$ and partially on the regression line $\kappa(c)=\operatorname{tg}(\alpha) c+\mathrm{q}_{1}$. Clearly, the regression line $\kappa(c)=\operatorname{tg}(\beta) c+q_{2}$, is the asymptote of $\kappa^{\prime \prime \prime}\left(c-c_{B}\right)$. In other words, the graph of $\kappa^{\prime \prime \prime}\left(c-C_{B}\right)$ consists of the linear part (between the origin and the point $\mathrm{B}$ ) and the nonlinear part (between points B and E), which are connected just at the point $\mathrm{B}$. To obtain the smooth transition from one part to another, the slopes of these two parts at the point $\mathrm{B}$ must be equal. Therefore, the following equality must be true:

$\operatorname{tg} \beta+(K / \tau)=\operatorname{tg}(\alpha)$

From (17) follows an important result for the ratio $(K / \tau)$ :

$\mathrm{K} / \tau=\operatorname{tg} \alpha-\operatorname{tg} \beta$

Fourthly, determine the point $\mathrm{B}\left(\mathrm{c}_{\mathrm{b}}, \mathrm{\kappa}_{\mathrm{b}}\right)$. From Fig. 5 it is clear that the point $B$ is a cross-section point of the straight lines $\kappa(\mathrm{c})=\operatorname{tg}(\alpha) \mathrm{c}+\mathrm{q}_{1}$ and $\kappa(\mathrm{c})=\operatorname{tg}(\beta) \mathrm{c}+\left(\mathrm{q}_{2}-\mathrm{K}\right)$. Note that though the Fig. 5 present the situation as if $q_{1}=0$, in gen- 
eral $\mathrm{q}_{1} \neq 0$. Therefore, in the following derivations we will consider $\mathrm{q}_{1} \neq 0$. After short manipulation we get the coordinates of the point $\mathrm{B}\left(\mathrm{c}_{\mathrm{B}}, \kappa_{\mathrm{B}}\right)$ :

$\mathrm{c}_{\mathrm{B}}=\left(\mathrm{q}_{2}-\mathrm{K}\right) /(\operatorname{tg} \alpha-\operatorname{tg} \beta), \kappa_{\mathrm{B}}=\operatorname{tg}(\alpha) \mathrm{c}_{\mathrm{B}}+\mathrm{q}_{1}$

Having this, we need to identify the origin of $\kappa^{\prime \prime}(\mathrm{c})$ with the point $B$. To do this we shift the $\kappa^{\prime \prime}(\mathrm{c})$ along the horizontal axis by $C_{B}$ units and then along the vertical axis by $\Delta=\operatorname{tg}$ $(\beta) c_{B}$ units. In this way we obtain the following analytic expression of the function $\kappa^{\prime \prime \prime}\left(\mathrm{c}-\mathrm{C}_{\mathrm{B}}\right)$ :

$\kappa^{\prime \prime \prime}\left(\mathrm{c}-\mathrm{c}_{\mathrm{B}}\right)=\kappa^{\prime \prime}\left(\mathrm{c}-\mathrm{c}_{\mathrm{B}}\right)+\Delta=\kappa^{\prime \prime}\left(\mathrm{c}-\mathrm{c}_{\mathrm{B}}\right)+\operatorname{tg}(\beta) \mathrm{C}_{\mathrm{B}}$

After taking into account (13) we finally obtain:

$\kappa^{\prime \prime \prime}\left(\mathrm{c}-\mathrm{c}_{\mathrm{B}}\right)=\operatorname{tg}(\beta)\left(\mathrm{c}-\mathrm{c}_{\mathrm{B}}\right)+\mathrm{K}\left[1-\exp \left(-\left(\mathrm{c}-\mathrm{c}_{\mathrm{B}}\right) / \tau\right]\right.$

$+\mathrm{d}+\operatorname{tg}(\beta) \mathrm{C}_{\mathrm{B}}$

Using denotation $\mathrm{D}=\mathrm{d}+\Delta=\mathrm{q}_{2}-\mathrm{K}+\operatorname{tg}(\beta) \mathrm{C}_{\mathrm{B}}$, the analytic expression of the searched approximating curve will be given by the equation:

$\kappa^{\prime \prime \prime}\left(c-c_{B}\right)=\operatorname{tg}(\beta)\left(c-c_{B}\right)+K\left[1-\exp \left(-\left(c-c_{B}\right)\right) / \tau\right]+D$

Finally, the complete approximation of the measured conductivity consists of the linear and nonlinear parts as it is expressed by the equation:

$\kappa(c)=\operatorname{tg}(\alpha) c+q_{1}$ if $c \leq c_{B}$ and $\kappa(c)=\kappa^{\prime \prime \prime}\left(c-C_{B}\right)$ ifc $\geq C_{B}$

The graph of $\kappa^{\prime \prime \prime}\left(\mathrm{c}-\mathrm{C}_{\mathrm{B}}\right)$ is drawn by the thick curve. As shows Fig. 5 the nonlinear function $\mathrm{K}^{\prime \prime \prime}\left(\mathrm{c}-\mathrm{C}_{\mathrm{B}}\right)$ is smoothly concatenated with the straight line $\kappa(c)=\operatorname{tg}(\alpha) c+q_{1}$ just at the point $B$.

\subsection{Optimization procedure}

The role of the optimization is to find optimal values of the parameters $\mathrm{K}$ and $\tau$, which will minimize a criterion of an optimal match of the function $\kappa^{\prime \prime \prime}\left(\mathrm{c}-\mathrm{C}_{\mathrm{B}}\right)$ with the samples of the conductivity $\kappa(\mathrm{c})$ obtained from the laboratory experiment. In the role of a measure of the optimal match is used the standard Root Mean Squared Error (RMSE).

Let the entries of a vector $\gamma$ represent the measured samples of the conductivity $\kappa(\mathrm{c})$ between points B and E. Let us recall that with respect to equation (18), once the value of the parameter $\mathrm{K}$ is known the point $\mathrm{B}$ is unambiguously determined. Moreover, since the slope of the function $\kappa^{\prime \prime \prime}\left(\mathrm{C}-\mathrm{C}_{\mathrm{B}}\right)$ at the point $\mathrm{B}$ and the slope $(\operatorname{tg} \alpha)$ of the first regressing line are equal, the parameter $\tau$ is also determined. Finally, let the entries of a vector $\hat{y}$ represent exact values of the function $\kappa^{\prime \prime \prime}\left(\mathrm{C}-\mathrm{C}_{\mathrm{B}}\right)$ corresponding to the homothetic entries of the vector y of the measured samples. Let the difference between vectors $y$ and $\hat{y}$ is the vector of the adaptation errors $\mathrm{e}=\mathrm{y}-\hat{\mathrm{y}}$. Then the standard RMSE criterion is given in the form:

$\operatorname{RMSE}=\sqrt{\frac{\mathrm{e}^{\mathrm{T} e}}{\mathrm{~N}}}$

where $\mathrm{N}$ is the length of the error vector e, $e^{T}$ is a transposition of $\mathrm{e}$ and $e^{T} e$ is a scalar product of the vector $e$. At this point we can apply any standard optimization procedure to find an optimal value of the parameter $\mathrm{K}$. In this case is used the downhill simplex algorithm as implemented in $P Y$ thon module SciPY.

\subsection{The corrected value of the $1^{\mathrm{st}} \mathrm{cmc}$}

The $1^{\text {st }} \mathrm{cmc}$ is commonly approximated by the c-coordinate of the point A. Hawing the optimal value of $\mathrm{K}$ and $\tau$ determined, one can insert them into $\kappa^{\prime \prime \prime}\left(\mathrm{c}-\mathrm{C}_{\mathrm{B}}\right)$ so as to obtain the optimal approximation of the measured conductivities.. Subsequently one can find a corrected value of the $1^{\text {st }} \mathrm{cmc}$ as a concentration at which the function $\kappa^{\prime \prime \prime}\left(c-C_{B}\right)$ exhibits the maximal curvature - CURV. Alternatively, the minimal Radius of curvature $=1 / C U R V$ ) can be also determined. As follows from the theory of analytic plane curves, the curvature CURV of the curve $\mathrm{\kappa}^{\prime \prime \prime}(\mathrm{c})$ can be calculated in accordance with the equation:

CURV $=\frac{\frac{\mathrm{d}^{2} \mathrm{~K}^{\prime \prime \prime}(\mathrm{c})}{\mathrm{dc}^{2}}}{\sqrt{\left(1+\left(\frac{\mathrm{d} \mathrm{K}^{\prime \prime \prime}(\mathrm{c})}{\mathrm{dc}}\right)^{2}\right)^{3}}}$

As to determination of an analytical expression for maximal value of the CURV may be the tedious task even for simple functions, it can be easily found by a suitable searching procedure. In this case was applied the linear search for finding a concentration value which maximizes the criterion CURV over the interval $\left(c_{B}-c_{E}\right)$. The theory that was derived so far is verified by the simulation experiment. The results are demonstrated and summarized in the following example.

\subsection{Example}

The conductivity of the solution of the surfactant hexadecylpyridinium bromide as a function of its concentrations at the temperature $30^{\circ} \mathrm{C}$ was obtained in the laboratory experiment [3]. The obtained samples are summarized in the Table 1 and the corresponding regression lines are shown in Fig. 6.

The optimal approximation of $\kappa(\mathrm{c})$-samples in the Table 1 is represented by the partially nonlinear function (23) and depicted in Fig. 7. The approximation was obtained by the downhill simplex algorithm.

The detailed view of the vicinity of the point $\mathrm{A}$ is shown in the Fig. 8 where is seen the magnified cutout of the rectangle

\begin{tabular}{|c|c|c|c|c|c|c|c|c|c|c|c|c|c|c|}
\hline $\begin{array}{c}\mathrm{c} \times 10^{4} \\
{\left[\mathrm{~mol} \cdot \mathrm{dm}^{-3}\right]}\end{array}$ & 1 & 2 & 3 & 4 & 5 & 6 & 7 & 8 & 9 & 10 & 11 & 12 & 13 & 14 \\
\hline $\begin{array}{c}\kappa \\
{\left[\mu \mathrm{S} \cdot \mathrm{cm}^{-1}\right]}\end{array}$ & 10.7 & 20.6 & 30.3 & 39.2 & 48.8 & 58.5 & 64.7 & 70.0 & 75.4 & 78.7 & 82.4 & 85.4 & 89.9 & 93.6 \\
\hline
\end{tabular}

Table 1 Conductivity of the solution of the surfactant hexadecylpyridinium bromide as a function of its concentrations at $30^{\circ} \mathrm{C}$ 
$(5.8-7.4) \times(55.0-70)$ around the point A. The concentration corresponding to the maximal value of the function CURV is denoted as the $1^{\text {st }} \mathrm{cmc}$ corrected. As can be seen this value is slightly lower than the intersection of the regression lines.

Contrary to the traditional static model (5) or IF - THEN model, there is suggested a dynamical model in the form of the $1^{\text {st }}$ order linear differential equation and optimal values of its parameters, namely the $\mathrm{K}_{\mathrm{opt}}$ and $\tau_{\text {opt }}$ are determined. The dependence $\kappa(\mathrm{c})$ is optimally (in the view of the RMSE criterion) approximated by the solution of the dynamical model.

The instantaneous gradient $\left.\left(\mathrm{d \kappa}^{\prime \prime \prime}\left(\mathrm{c}-\mathrm{c}_{\mathrm{B}}\right)\right) / \mathrm{dc}\right)$ was also evaluated. In Fig. 9 it is given by the doted curve. The scale of the gradient is appended on the right side of the figure. The gradient is constant up to $\mathrm{C}_{\mathrm{B}}$. It indicates that in the solution is present a sufficient number of the free ions and consequently, the conductivity increases proportionally to the concentration. But for the concentrations $C>C_{B}$ the gradient nonlinearly decreases. The reason is that the number of micelles increases, which hampers of the movability of free ions. Hence, for concentrations $c>c_{B}$ the gradient serves as a measure of the intensity of creation of micelles or a measure of the process activity.

\section{Conclusions}

Every natural process is governed by laws of nature and as such it runs smoothly and (in general) nonlinearly. The sharp jumps of its characteristic quantities may be observed exclusively on its more or less (in)accurate model. This phenomenon is also observed in the behavior of the electrical conductivity of the surfactant solution as a measure of a number of the micellized surfactant molecules. That is why the process of

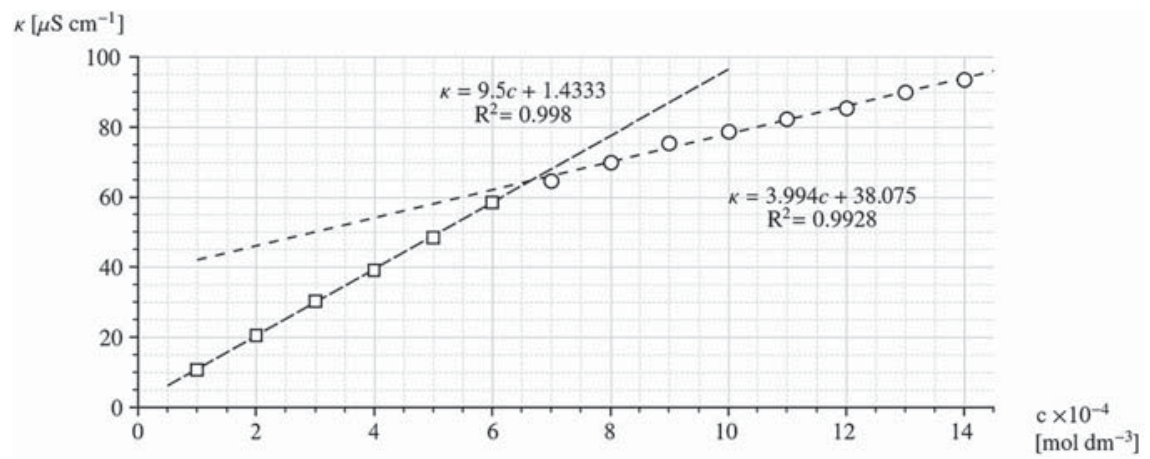

$\kappa\left[\mu \mathrm{S} \mathrm{cm}^{-1}\right]$

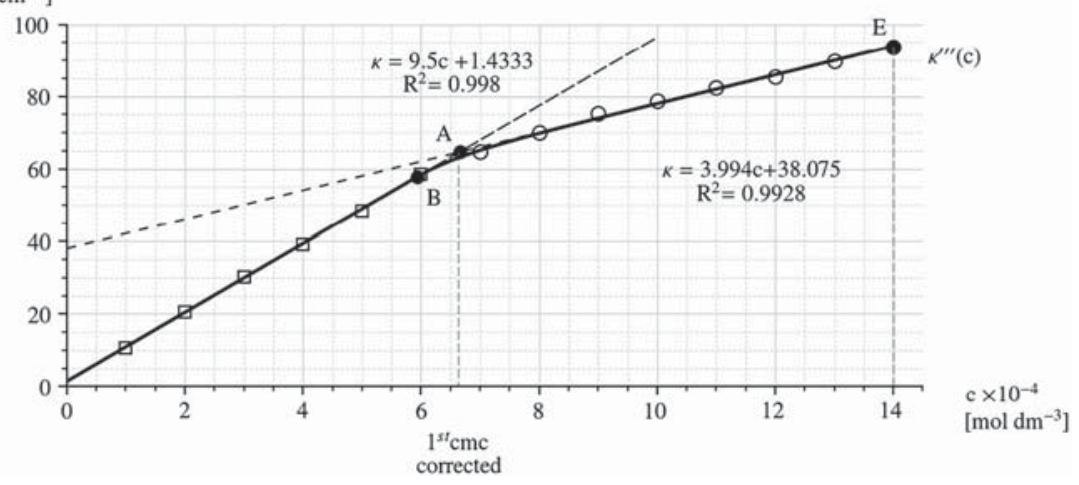

Figure 6 The dependence $\kappa(\mathrm{c})$ obtained by the experimental measurements
Figure 7 The optimal dependence $\kappa(c)$. Experimental samples (squares and circles), optimized approximation by dynamic model (thick curve between points $B$ and $E$ )

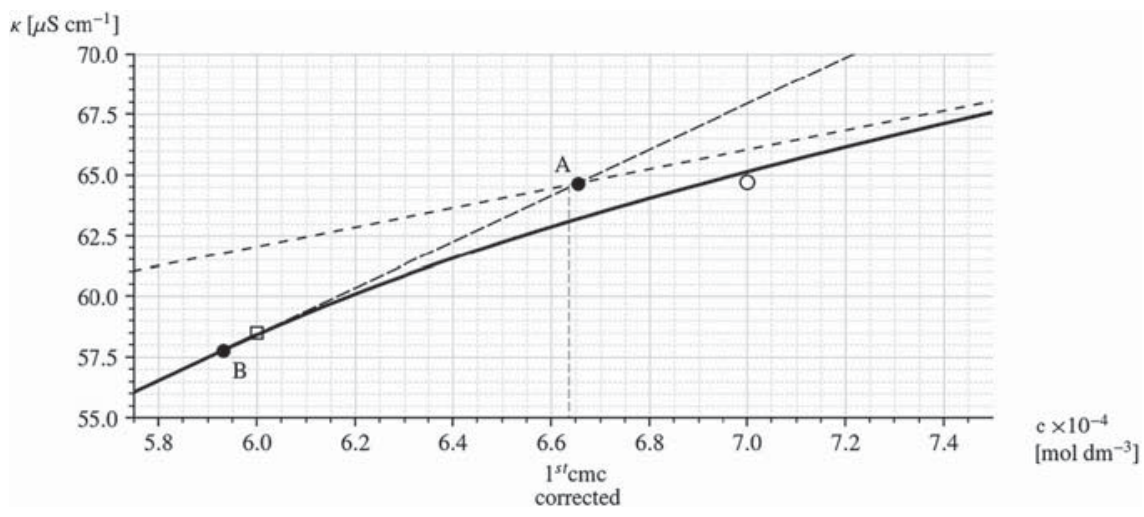

Figure 8 Magnified vicinity of the point A with the corrected value of the $1^{\text {st }} \mathrm{cmc}$ 


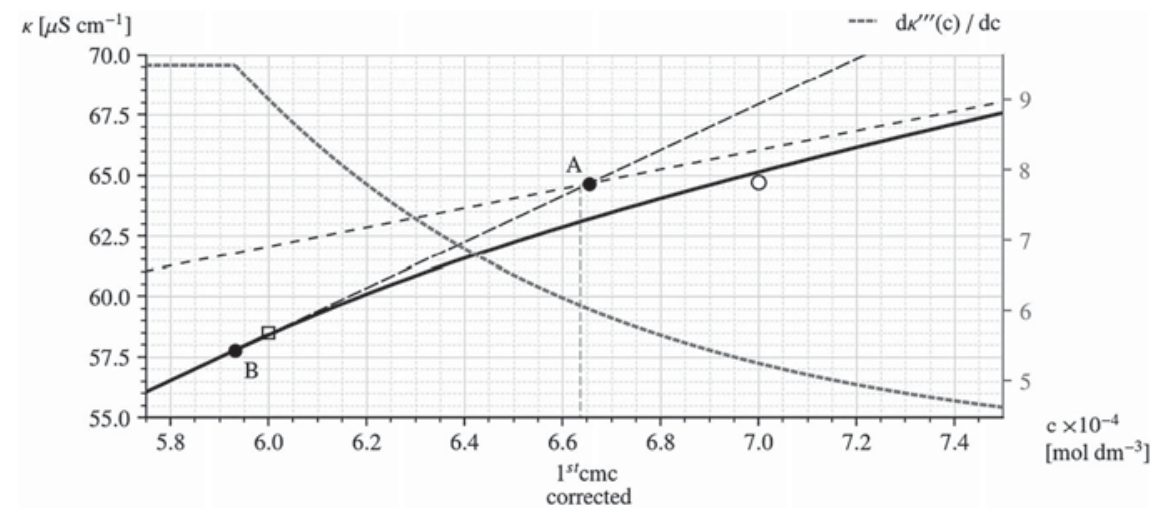

Figure 9 The vicinity of the $1^{\text {st }} \mathrm{cmc}$ with the gradient $\left.d \kappa^{\prime \prime \prime}\left(c-c_{B}\right)\right) / d c$ micellization should be considered to be a dynamical process.

The analytical expressions for two traditional static models based on the interval description of the dependence of the conductivity vs. concentration were presented. The first is expressed by the Heaviside unit functions, while the other uses IF-THEN rules.

The dynamical model of the process of micellization reflecting the dynamics of the natural processes was suggested in the form of the $1^{\text {st }}$ order linear differential equation.

The model was a stepping stone for derivation of the theoretical basis of the optimal nonlinear approximation of the measured $\kappa(\mathrm{c})$-samples. As a result the analytical expression of the optimal approximation curve was obtained. The complete approximation function consists of two parts, namely the linear regressing straight line and the optimal nonlinear function. The optimal point (the point B) where these two partial functions are to be concatenated was determined.

Due to the evaluation of the maximal curvature of the nonlinear part it was possible to find an optimal value of the $1^{\text {st }} \mathrm{cmc}$. The procedure was verified by the realistic example and the following optimal values were obtained:

$\mathrm{B}_{\text {opt }}(5.932,57.785)$

$\mathrm{K}_{\text {opt. }}=3.98$

$\tau_{\mathrm{opt}}=0.72$

$\operatorname{maxCURV}=0.0123288$

$1^{\text {st }} \mathrm{cmc}=6,655 \times 10^{-4}\left[\mathrm{~mol} \mathrm{dm}{ }^{-3}\right]-$ determined by the measurement

$1^{\text {st }} \mathrm{cmc}$ corrected $=6.636 \times 10^{-4}\left[\mathrm{~mol} \mathrm{dm}{ }^{-3}\right]-$ determined by the concentration in which max CURV is reached

As the results say, values of the $1^{\text {st }} \mathrm{cmc}$ and the $1^{\text {st }} \mathrm{cmc}$ corrected are close to each other. The difference is prevailingly caused by using the point A (determined by pure linear regression) as a main carrier of information about the $1^{\text {st }} \mathrm{cmc}$.

The salient feature of the presented dynamic modelling and the nonlinear approximation following from it, is that the approximation of the measured samples is much close to the reality than pure linear approximation by straight regression lines.

\section{Acknowledgements}

The paper is one of the outcomes of the research work for the project ntitled "Research center for severe diseases and related complications", ITMS: 26240120038. This project is co-financed by the European Union "We support research activities in Slovakia".

\section{References}

1. Muller P.: Glossary of terms used in physical organic chemistry. UPAC recommendations 1994, Pure \& Appl. Chem, 66 (1994), 1077- 1184. DOI: 10.1351/pac199466051077

2. Moroi, Y.: Micelles: Theoretical and applied aspects. Plenum press., New York, 1992, ISBN 0-30643996-4.

3. Oremusová, J., Vitková, Z. and Vitko, A.: Study of micelle properties and thermodynamics of micellization of the Benzethonium chloride. Tenside Surf. Det. 49 (2012), 4, 322-329. DOI: 10.3139/113.110198

4. González-Pérez, A., Czapkiewicz, J., Prieto, G. and Rodríguez, J. R.: Second critical micelle concentration of dodecyldimethyl benzylamminium chloride in aqueous solution at $25^{\circ}$ C; Colloid Polym Sci 281 (2003), $1191-1195$,. DOI:10.1007/s00396-003-0905-2

5. Savaroglu, G. and Yurt, A.: Determination of the second critical micelle concentration of benzyldimethyltridecylazanium chloride in aqueous solution by acoustic and conductometric measurements. J. Chem. Thermodynamics 43 (2011). DOI:10.1016/j.jct.2011.05.011

6. Garcia-Mateos, I., Mercedes Velazquez, M. and Rodriguez, L. J.: Critical micelle concemtration determination in binary mixtures of ionic surfactants by deconvolution of conductivity/concentration curves. Langmuir 6 (1990), 10781083. DOI:10.1021/la00096a009

7. López Fontán, J. L., Costa, J., Ruso, J. M., Prieto, G. and Sarmiento, F.: A nonparametric approach to calculate critical micelle concentration: The local polynomial regression method. Eur. Fyz, J. 13 (2004)133 - 140. PMid:15052423; DOI:10.1140/epje/e2004-00050-3

8. Sungwon, K., Yunzhou, S., Ji, Y. K., Kinam, P. and Ji-Xin, C.: Overcoming the barriers in micellar drug delivery: loading efficiency, in vivo stability, and micelle-cell interaction. Journal Expert Opinion on Drug Delivery. 7 (2010), 49 62. PMid:20017660; DOI:10.1517/17425240903380446

9. Vitková, $Z$. et al.: Pharmacokinetic and pharmacodynamics from the perspective of pharmaceutical technology. (In-silico approach) (in Slovak). Felia S.r.o. Bratislava, (2016) ISBN.978-80-89824-03-8.

10. Vitková, Z., Tárník, M., Miklovičová, E., Murgaš, J., Oremusová, J. and Vitko, A. System-based approach to prediction of surfactants' influence on pharmacokinetics and pharmacodynamics. Tenside Surf. Det. 57 (2020), 1 - 7. DOI:10.3139/113.110661

11. Driels M.: Linear control systems engineering. McGraw-Hill 1996, ISBN 0-07017824-0.

12. Al-Sufi, W., Pineiro, L. and Novo, M.: A model for monomer and micellar concentrations in surfactant solutions: Application to conductivity, NMR, diffusion, and surface tension data. J. Colloid and Interface Sci. 370 (2012), 102- 110. PMid:22265231; DOI: 10.1016/j.jcis.2011.12.037

Received: 24.07 .2020

Accepted: 04.10.2020

Bibliography

DOI 10.1515/tsd-2020-2296

Tenside Surf. Det. 58 (2021) 1, page 4- 12

(c) 2021 Walter de Gruyter GmbH, Berlin/Boston, Germany

ISSN 0932-3414 · e-ISSN 2195-8564

\section{Correspondence address}

Dr. Marián Tárník

Institute of Robotics and Cybernetics

Faculty of Electrical Engineering and Information Technology

Ilkovičova 3

81219 Bratislava

Slovak Republic

E-Mail: marian.tarnik@stuba.sk 


\section{The authors of this paper}

Eng. Jarmila Oremusová, PhD. graduated from the Faculty of Chemical Technology of the Slovak University of Technology in Bratislava. Research: Physical-chemical analysis of drugs.

Assoc. Prof. Dr. rer. nat. Zuzana Vitková, PhD. graduated from the Faculty of Pharmacy of the Comenius University in Bratislava, where she has been acting as a teacher and researcher till 2018. At present she is with the Bio-cybernetic group of the Institute of Robotic and Cybernetics, Slovak University of Technology in Bratislava. Research: Drug delivery, bio-pharmacy, pharmacokinetics, pharmacodynamics

Prof. Eng. Jarmila Pavlovičová, PhD. graduated from the Faculty of Electrical Engineering andInformation Technology of the Slovak University of Technology in Bratislava. Research: Softcomputing, intelligent systems and bio-cybernetics.
Prof. Eng. Anton Vitko, PhD. graduated from the Faculty of Electrical Engineering and Information Technology of the Slovak University of Technology in Bratislava. Research: Measurements and control of dynamic systems, bio-cybernetics

Assoc. Prof. Andrej Babinec, PhD. graduated from the Faculty of Electrical Engineering and Information Technology of the Slovak University of Technology in Bratislava. Research: Theory of automatic control, robotics

Eng. Marian Tárnik, PhD. graduated from the Faculty of Electrical Engineering and Information Technology of the Slovak University of Technology in Bratislava. Research: Theory of automatic control, bio-cybernetics, intelligent systems

Prof. Eng. Ján Murgaš, PhD. graduated from the Faculty of Electrical Engineering and Information Technology of the Slovak University of Technology in Bratislava. Research: Theory of automatic control, intelligent systems and bio-cybernetics. 